\title{
SYNONYMY IN DEVELOPING TERM SYSTEMS (USING THE EXAMPLE OF THE TERM MOBBING IN MODERN ENGLISH)
}

\author{
Olga A. Bursina \\ Vologda State University, Vologda, Russia
}

\begin{abstract}
The paper elucidates the reasons for terminology synonymy. The example of the term mobbing is shown, that this phenomenon is especially characteristic of new developing term systems. It is revealed that the term mobbing belongs to several related term systems and has a number of synonyms. The following reasons for terminology synonymy are observed in the work: terms polysemy, introduction of a term by different scholars and publicists in different periods and countries, the ability of a term to have a broader meaning, enlargement of the form of a term.

The three main components of the meaning of the term mobbing given in the scientific and reference literature are distinguished as a result of the definition analysis. In case the semantic structure of other terms nominating this social psychological phenomenon comprises these components, one can state that they are synonymous. It is proved in the paper that some terms which are considered to be synonyms of the term mobbing in specialist literature are actually not its synonyms. The semantic analysis of the synonymic row with the head term mobbing and the analysis of their semantic structure prove that it is necessary to regulate the micro term system Mobbing.

Key words: term, terms' synonymy, term system, term microsystem, context, term's semantics, term structure, synonymic row.

Citation. Bursina O.A. Synonymy in Developing Term Systems (Using the Example of the Term Mobbing in Modern English). Vestnik Volgogradskogo gosudarstvennogo universiteta. Seriya 2, Yazykoznanie [Science Journal of Volgograd State University. Linguistics], 2018, vol. 17, no. 1, pp. 143-150. (in Russian). DOI: https:// doi.org/10.15688/jvolsu2.2018.1.15
\end{abstract}

\section{СИНОНИМИЯ В ФОРМИРУЮЩИХСЯ ТЕРМИНОСИСТЕМАХ (НА ПРИМЕРЕ ТЕРМИНА МОВВING В СОВРЕМЕННОМ АНГЛИЙСКОМ ЯЗЫКЕ)}

\section{Ольга Алексеевна Бурсина}

Вологодский государственный университет, г. Вологда, Россия

\begin{abstract}
Аннотация. Статья посвящена проблемам возникновения синонимии в современных терминологических системах. На примере термина mobbing показано, что синонимические отношения характерны для формирующихся терминосистем. Установлено, что термин mobbing входит в несколько терминосистем смежных областей знания и имеет большое количество синонимов. В качестве причин появления у термина $\infty$ mobbing синонимов определены следующие: возникновение терминологической многозначности; введение 훙 термина учеными и публицистами разных стран в разное время; способность термина обозначать широкие i понятия; усложнение формальной структуры термина.

В результате дефиниционного анализа определений термина mobbing, приведенных в научной и справочной литературе, выделены три основных компонента его значения. Их наличие в семантике других единоц, которые номинируют исследуемый социально-психологический феномен, является критерием для установления отношений синонимии между терминами. В работе показано, что некоторые термины, квалифи(2) цированные в научной литературе как синонимы термина mobbing, таковыми не являются. В результате
\end{abstract}


семантического анализа синонимического ряда во главе с термином mobbing и анализа структуры единиц, входящих в него, делается вывод о необходимости упорядочения микротерминосистемы Mobbing.

Ключевые слова: термин, терминологическая синонимия, терминосистема, микротерминосистема, контекст, семантика термина, структура термина, синонимический ряд.

Цитирование. Бурсина О. А. Синонимия в формирующихся терминосистемах (на примере термина mobbing в современном английском языке) // Вестник Волгоградского государственного университета. Серия 2, Языкознание. -2018. - Т. 17, № 1. - C. 143-150. - DOI: https://doi.org/10.15688/jvolsu2.2018.1.15

\section{Введение}

Проблема синонимии терминов вызывает дискуссии среди лингвистов, оставаясь актуальной на протяжении долгого времени. В научном сообществе аккумулируется все больше практического и теоретического знания относительно специальной лексики. На фоне меняющейся научной картины мира терминологическую синонимию не следует рассматривать как недопустимое в терминосистеме лексико-семантическое явление. Практика показывает, что существует большое количество терминовсинонимов, особенно в формирующихся терминосистемах.

В условиях быстрого и большого потока информации появляются новые области научно-технического знания и, соответственно, зарождаются и развиваются новые терминосистемы. Некоторые ученые утверждают, что терминологическая синонимия - признак не зарождающейся, a, напротив, бурно развивающейся науки. Так, В.А. Татаринов отмечает, что «чем выше уровень развития науки, тем синонимичнее мышление специалиста» [Татаринов, 1996, с. 227]. Однако большинство исследователей, и мы разделяем их мнение, склонны считать, что наличие лексико-семантических отношений между терминологическими единицами, в частности отношений синонимии, особенно характерно для терминосистем на этапах их формирования (см., например: [Гринев-Гриневич, 2008; Даниленко, 1977; Загоровская, 2011; Ткачева, 1987]).

Терминосистемы социально-гуманитарных наук имеют свою специфику. (см., например: [Лейчик, 2006; Цвиллинг, 1989]). Они интересны для изучения с методологической точки зрения, так как «отличаются обобщенностью, тесной связью с общеупотребительной лексикой и терминосистемами смежных дисциплин, некоторой смысловой разветвленностью, отсутствием унифицированности, неустойчивостью значений, эмоциональной и субъективно-оценочной окраской, контекстной зависимостью, стилистической окраской, ограниченностью форм, не экономичностью, нередко отсутствием научного обоснования» [Бурсина, 2010, с. 98]. Перечисленные особенности терминосистем социально-гуманитарного направления способствуют развитию лексико-семантических отношений среди терминов, в том числе отношений синонимии.

В данной работе рассматривается синонимический ряд во главе с термином mobbing в формирующейся и развивающейся микротерминосистеме Mobbing, которая относится к области социально-гуманитарных знаний.

Моббинг как социально-психологическое явление появился гораздо раньше, чем привлек к себе общественный и научный интерес (в странах Западной Европы и США этот феномен обсуждается в обществе и исследуется учеными с начала 1980-х гг., в последние десятилетия проблема моббинга получила широкий общественный резонанс и в России).

В настоящее время данная проблема привлекает внимание ученых разных областей знания и практической деятельности: психологии, социальной работы, права, экономики, образования и др., поэтому термин mobbing входит в разные терминосистемы. Человек является членом коллектива или группы, начиная с детских лет и всю взрослую жизнь (группа детского сада, школа, детский дом, университет, общежитие, армия, трудовой коллектив, социальные сети). Такое широкое экстралингвистическое предметно-понятийное терминологическое поле (область или контекст) предполагает наличие соответствующего лингвистического терминологического аппарата, который должен представ- 
лять собой целостную микротерминосистему, используемую для описания проблемы моббинга и поиска возможных путей ее решения.

\section{Происхождение и употребление термина mobbing}

С лингвистической точки зрения важно проследить возникновение термина mobbing, который происходит от английского глагола to $m o b$ - «to crowd about and attack or annoy» (MWOD). C.A. Дружилов отмечает, что термин mobbing был введен в 1958 г. К. Лоренцем и обозначал феномен группового нападения нескольких мелких животных на более крупного противника. Термин получил известность в 60-х гг. XX в. после публикации работы шведского врача П. Хайнемана, который сравнивал жестокое поведение детей по отношению к сверстникам с агрессивным поведением животных. В современном значении термин впервые употребил практический психолог и инженер-экономист Г. Лейман, изучавший в начале 80-х гг. XX в. особенности поведения людей на работе, в трудовом коллективе [Дружилов, 2011, с. 75].

Обратимся к дефиниции термина, предложенной доктором Г. Лейманом:

mobbing - hostile and unethical communication, which is directed in a systematic way by one or a few individuals mainly towards one individual who, due to mobbing, is pushed into a helpless and defenceless position, being held there by means of continuing mobbing activities (Leymann).

Данное определение является основой более современных дефиниций, предлагаемых новейшими англоязычными словарями и энциклопедиями. Например, краткая энциклопедия по сестринскому делу под ред. Г. Фельдмана, изданная в 2011 г., содержит следующую дефиницию:

mobbing - an aggressive antagonistic behavior implemented by a group of people toward one or more individuals that aims to humiliate, discredit, and marginalize a targeted individual (Feldman).

Русскоязычные толковые и специальные словари приводят определения моббинга, аналогичные процитированным. Например, толковый словарь русского языка, изданный в
2011 г. под ред. Т.Ф. Ефремовой, дает следующее определение:

моббинг - психологический террор, интриги в отношении того, кто может составить конкуренцию (в условиях рыночной экономики) (Ефремова).

В учебном словаре терминов рекламы и паблик рилейшенз 2007 г. находим более развернутое определение:

моббинг - (от англ. mobbing) несправедливое отношение (создание для работника заведомо невыгодных условий) к работнику, издевательство над ним или его дискриминация со стороны работодателя или других работников (Радченко).

В научных работах по психологии предлагается следующая дефиниция:

моббинг - (mobbing - от англ. глагола to mob грубить, нападать толпой, стаей, травить) - форма психологического насилия в виде травли сотрудника в коллективе с целью его последующего увольнения; проявляется в виде психологических притеснений работника, происходящих на протяжении длительного времени и включающих в себя негативные высказывания, необоснованную критику в адрес работника, его социальную изоляцию, распространение о работнике заведомо ложной информации и т. п. (Дружилов, с. 119).

Научное сообщество однозначно понимает феномен моббинга, именно поэтому термины mobbing и моббинг имеют схожие дефиниции, в которых актуализируются идентичные компоненты значения:

- 'что предпринимается': hostile and unethical communication / an aggressive antagonistic behavior / психологический террор / несправедливое отношение, издевательство, дискриминация / форма психологического насилия;

- 'кем и в отношении кого': by one or a few individuals mainly towards one individual / by a group of people toward one or more individuals / $\kappa$ работнику со стороны работодателя или других работников / сотрудника в коллективе;

- 'каков результат': is pushed into a helpless and defenceless position / aims to humiliate, discredit, and marginalize / с иелью его последующего увольнения. 


\section{Причины}

\section{терминологической синонимии}

В терминосистеме социальной работы, а также в смежной терминосистеме психологии, существуют и другие термины, обозначающие моббинг как социальный феномен. Например, Л. Крошоу в одной из своих работ говорит о беспорядочном наименовании данного феномена различными зарубежными исследователями и приводит 33 термина, которые используются для называния этого явления: abuse, abusiveness, aggression, bullying, bullying / mobbing, counterproductive workplace behavior, emotional abuse, emotional harassment, employee emotional abuse, generalized workplace abuse, harassment, hostile workplace behavior, maltreatment, mistreatment, mobbing, nonphysical aggression, nonsexual harassment, non-status-based harassment, psychological abuse, psychological aggression, psychological harassment, psychological terror, scapegoating, status-blind bullying, status-conscious bullying, unlawful bullying, vexatious behavior, workplace abuse, workplace aggression, workplace harassment, workplace hostility, workplace incivility, workplace psychological violence [Crawshaw, 2009, p. 264].

Итальянские исследователи также отмечают, что в разных странах для обозначения рассматриваемого общественного феномена используются разные термины: bullying, work or employee abuse, mistreatment, emotional abuse, bossing, victimization, intimidation, psychological terrorization, psychological violence [Cassitto et al., 2003, p. 12]. Однако, по их мнению, данные термины не являются полными синонимами, поскольку каждый из них возник в том или ином культурном контексте и имеет свою специфику.

Так, термин bullying изначально использовался применительно к школьной среде, обозначая преследование члена школьного коллектива. В настоящее время термин приобрел более широкое значение и имеет следующую дефиницию:

bullying - intentional harm-doing or harassment that is directed toward vulnerable targets and typically repeated. Bullying encompasses a wide range of malicious aggressive behaviours, including physical violence, verbal mockery, threats, ostracism, and rumours spread either orally or by other means of communication, such as the Internet (Britannica).

Соответственно, явление, обозначаемое данным термином, может наблюдаться не только в школьном, но и в рабочем коллективе, в семье, в любой другой группе людей (соседей, прихожан церкви и т. д.). Развитие значений термина bullying отражает развитие терминосистемы, в процессе которого входящие в нее термины могут вступать в отношения семантического сходства и, как следствие, входить в синонимические ряды. Итак, формирование многозначности - это первая причина терминологической синонимии. Однако многозначность может быть снята в случае, если усложняется структура термина. Несомненно, двухкомпонентный термин workplace bullying отличается от однокомпонентного bullying как более узким значением, так и принадлежностью к разным микротерминосистемам.

Второй причиной образования синонимичных терминов может быть место и время появления того или иного термина. Например, немецкий ученый, психолог, психиатр Г. Лейман в 1980-х гг. основал специализированную клинику в Швейцарии и впервые провел исследования психологического насилия в виде травли сотрудника в коллективе, назвав его mobbing. Синонимичный ему термин workplace bullying был введен в 1992 г. британской журналисткой Андреа Адамс, которая вынесла проблему моббинга на широкое общественное обсуждение. В 1998 г. Доктор Р. Нами и социальный психолог, основатель и консультант образовательного и исследовательского института по вопросам моббинга Г. Нами начали использовать термин workplace bullying в американской прессе. Данный термин имеет следующее определение:

workplace bullying - repeated, health-harming mistreatment of one or more persons (the targets) by one or more perpetrators: abusive conduct that takes one or more of the following forms: verbal abuse, or threatening, intimidating behaviors (including nonverbal), or work interference - sabotage - which prevents work from getting done, or some combination of one or more (Namie). 
Дефиниция термина workplace bullying эксплицирует основные компоненты значения, аналогичные компонентам термина mobbing: 'что предпринимается' (persistent aggressive or unreasonable behavior, verbal, nonverbal, psychological, physical abuse and humiliation), 'кем и в отношении кого' (individuals or groups against a co-worker). Таким образом, синонимия терминов действительно может иметь место на этапе формирования терминосистемы в силу того, что ученые разных стран стремятся в один и тот же период назвать и определить то или иное явление или предмет.

Третья причина терминологической синонимии - широкое значение термина в силу достаточно широкого объема понятия, им номинированного. В список синонимов, составленный Л. Крошоу, входит термин abuse, который имеет следующее определение:

abuse - improper behavior intended to cause physical, psychological, or financial harm to an individual or group (цит. по: [Бурсина, 2016, с. 54]).

В дефиниции выражены три компонента значения, характерные для термина mobbing: 'что предпринимается' (improper behavior), 'кем и в отношении кого' (an individual or group), 'каков результат' (physical, psychological, or financial harm). Однако терминологическое значение настолько общее, что полностью совпадает с общелитературным лексическим значением данной единицы. При отсутствии необходимого контекста невозможно утверждать, что термины mobbing и abuse являются абсолютными синонимами.

Четвертая причина развития терминологической синонимии - усложнение структуры термина.

Л. Крошоу и ее итальянские коллеги в списке синонимов термина mobbing приводят термины emotional abuse, employee emotional abuse, generalized workplace abuse, psychological abuse, workplace abuse, work or employee abuse. Однако усложнение структуры термина abuse, способствующее уточнению его семантики, по-разному отражается на процессе образования синонимов. Так, термины (generalized) workplace abuse, work or employee abuse, psychological abuse, coдержащие в своем значении компоненты ('что предпринимается', 'кем и в отношении кого', 'каков результат'), являются полными синонимами и принадлежат микротерминосистеме Mobbing:

(generalized) workplace / work / employee abuse - physical violence or harassment that is physical in nature, such as inappropriate touching, threats, bullying, or verbal harassment; often, any behavior that causes a worker to feel attacked or unsafe (Namie)

psychological abuse - psychological abuse is a pattern of aggressive acts, verbal or non-verbal, intended to harm another person mentally or emotionally and/or to exert control over that person (Breiding).

Однако термин emotional abuse не является абсолютным синонимом термина mobbing. Во-первых, он принадлежит хотя и смежным, но самостоятельным терминосистемам (социальная работа, психология, медицина и психиатрия), имея в них свое специальное значение. Во-вторых, даже в пределах одной терминосистемы он может входить в несколько микротерминосистем. Например, внутри терминосистемы области социальной работы термин emotional abuse входит в микротерминосистемы "Mental health, substance abuse and social work", "Family, children, education, old people and social work", имея в них следующие дефиниции:

the debasement of a person's feelings that causes the individual to perceive himself or herself as inept, not cared for, and worthless (MMD);

the third most frequently reported form of child abuse; includes acts or omissions by the parents or other caregivers that could cause serious behavioral, emotional, or mental disorders. For example, the parents/caregivers may use extreme or bizarre forms of punishment, such as confinement of a child in a dark closet (Loveleen, p. 3).

Термин employee emotional abuse может быть включен в исследуемый синонимический ряд во главе с термином mobbing, поскольку имеет в своей структуре дополнительный компонент (employee). В определении данного термина отражены все три компонента значения, выявленные у термина mobbing: 
employee emotional abuse - a repetitive, targeted, and destructive form of communication directed by more powerful members at work at those less powerful (Lutgen-Sandvik).

Расширение формальной структуры термина ведет к сужению, специализации его значения. Данное утверждение верно и для таких терминов, как aggression / workplace aggression, harassment / workplace harassment. Каждый структурный элемент привносит дополнительные компоненты в семантику термина, тем самым уточняя его, но не способствует преодолению синонимии.

\section{Выводы}

Отношения синонимии характерны для специальной лексики, в частности на этапе формирования терминосистем. На примере семантического анализа термина mobbing показано, что в процессе формирования микросистемы терминов основной задачей специалистов является упорядочение терминов, исключение из оборота терминов, семантика которых вне контекста представляется неясной и нечеткой, а также терминов, которые уже входят в данную или смежную систему, обозначая то или иное понятие. В качестве причин появления у термина mobbing синонимов были установлены: возникновение терминологической многозначности; введение термина учеными и публицистами разных стран в разное время; способность термина обозначать широкие понятия; усложнение формальной структуры термина.

\section{СПИСОК ЛИТЕРАТУРЫ}

Бурсина О. А., 2010. Специфика терминосистем социально-гуманитарных и естественно-математических наук // Вестник Ленинградского государственного университета имени А.С. Пушкина. Серия Филология. Т. 1, № 1. С. 92-99.

Бурсина О. А., 2016. Особенности гиперо-гипонимических отношений в отраслевых терминосистемах (на примере терминосистемы социальной работы в современном английском языке) // Вестник Вологодского государственного университета. Серия: Гуманитарные, общественные, педагогические науки. № 1. С. 53-56.

Гринев-Гриневич С. В., 2008. Терминоведение. М. : Академия. $304 \mathrm{c}$.
Даниленко В. П., 1977. Русская терминология. М. : Наука. 246 c.

Дружилов С. А., 2011. Моббинг на кафедре вуза в условиях реформирования высшего профессионального образования // Мир науки, культуры, образования. № 3. С. 74-77.

Загоровская О. В., 2011. Термин и терминология : монография. Воронеж : Научная книга. $146 \mathrm{c}$.

Лейчик В. М., 2006. Терминоведение: предмет, методы, структура. М. : ДомКнига. 256 с.

Татаринов В. А., 1996. Теория терминоведения. В 3 т. Т. 1. Теория термина: история и современное состояние. М. : Моск. лицей. $311 \mathrm{c}$.

Ткачева Л. Б., 1987. Основные закономерности английской терминологии. Томск : Изд-во Томск. ун-та. 200 с.

Цвиллинг М. Я., 1989. Специфика общественно-научного текста (к вопросу о внутриотраслевой дифференциации языка науки) // Разновидности и жанры научной прозы. Лингвостилистические особенности. М. : Наука. С. 27-36.

Cassitto M., Fattorini E., Gilioni R., Rengo C., 2003. Raising Awareness of Stress at Work in Developing Countries // Protecting Workers: Health Series. № 6. Milano : World Health Organisation. P. 1-38. URL: http://www. wascorporation.it/docs/psicological_stress.pdf (date of access: 15.02.2018).

Crawshaw L., 2009. Workplace Bullying? Mobbing? Harassment? Distraction by a thousand definitions // Consulting Psychology Journal: Practice and Research. Vol. 61, № 3. P. 263-267. URL: http://www.bosswhispering.com/CPJ\% 20Crawshaw\%20Article.pdf (date of access: 07.07.2017).

\section{ИСТОЧНИКИ И СЛОВАРИ}

Дружилов - Дружилов С. А., 2011. Проблемы моббинга на кафедре в условиях реформирования вуза // Высшее образование в России. № 6. C. 118-122. URL: http://druzhilov.ru/articles/ психологический-террор-боссинг-и-мо/.

Ефремова - Ефремова Т. Ф. Современный толковый словарь русского языка. URL: http://dic. academic.ru/dic.nsf/efremova/276686/моббинг.

Радченко - Радченко И. А., 2007. Учебный словарь терминов рекламы и паблик рилейшенз. Воронеж : ВФ МГЭИ. URL: http://advertising pr.academic. ru/275/Моббинг.

Breiding - Breiding M., Basile K., Smith S., Black M., Mahendra R., 2015. Intimate partner violence surveillance: Uniform definitions and recommended data elements, version 2.0. Atlanta, (GA) : National Center for Injury Prevention and Control, Centers 
for Disease Control and Prevention. 156 p. URL: https://www.cdc.gov/violenceprevention/pdf/ intimatepartnerviolence.pdf.

Britannica - Britannica. URL: https:/www.britannica. com/topic/bullying\#ref1 137234 (date of access: 07.07.2017).

Feldman-Nursing Leadership. A Concise Encyclopedia / ed. by Harriet R. Feldman. URL: http://www. omnilexica.com/?q=mobbing\#noun.

Leymann - Leymann H., 1990. Mobbing and Psychological Terror at Workplaces // Violence and Victims. № 5. P. 119-126. URL: http://www. mobbingportal.com/LeymannV\%26V1990(3).pdf (date of access: 07.07.2017).

Loveleen - Loveleen K., Varadan S., Kumar P., 2007. Study on Child Abuse: India 2007. Ministry of Women and Child Development, Government of India. New Delhi : Kriti. 192 p.

Lutgen-Sandvik - Lutgen-Sandvik P., 2003. The Communicative Cycle Of Employee Emotional Abuse. Generation and Regeneration of Workplace Mistreatment // Management Communication Quarterly. Vol. 16, № 4. P. 471501. URL: https://www.researchgate.net/ publication/258170720_The_Communicative Cycle_Of_Employee_Emotional_Abuse Generation_and_Regeneration_of_Workplace_ Mistreatment (date of access: 20.06 .2017 ).

MMD-Mosby's Medical Dictionary, 8th edition. 2009. URL: https://medical-dictionary.thefreedictionary. com/emotional+abuse (date of access: 15.02.2018).

MWOD - Merriam-Webster online dictionary. URL: https://www.merriam-webster.com/dictionary/ mob (date of access: 07.07.2017).

Namie - Namie G., Yamada D. C. Healthy Workplace Bill. URL: http://healthyworkplacebill.org/ problem/ (date of access: 15.02.2018).

\section{REFERENCES}

Bursina O.A., 2010. Specific character of the terminologies in Arts and Sciences. Vestnik Leningradskogo gosudarstvennogo universiteta imeni A.S. Pushkina. Seriya Filologiya [Vestnik of Pushkin Leningrad State University. Series. Philology], vol. 1, no. 1, pp. 92-99.

Bursina O.A., 2016. Typical features of hyperonymhyponym relations in terminologies (based on the example of social work terminology in modern English). Vestnik Vologodskogo gosudarstvennogo universiteta. Seriya: Gumanitarnye, obshchestvennye, pedagogicheskie nauki, no. 1, pp. 53-56.

Grinev-Grinevich S.V., 2008. Terminology studies. Moscow, Akademiya Publ. 304 p.
Danilenko V.P., 1977. Russian terminology. Moscow, Nauka Publ. 246 p.

Druzhilov S.A., 2011. Mobbing at a university's department in the conditions of high school reformation. Mir nauki, kultury, obrazovaniya, no 3, pp. 74-77.

Zagorovskaya O.V., 2011. Term and terminology studies. Voronezh, Nauchnaya kniga Publ. 146 p.

Leychik V.M., 2006. Terminology studies: subject, methods, structure. Moscow, DomKniga Publ. $256 \mathrm{p}$.

Tatarinov V.A., 1996. Terminology theory. In 3 vols. Vol. 1. Term theory: history and present. Moscow, Moscovsky Litsey Publ. 311 p.

Tkacheva L.B., 1987. Main tendencies in English terminology. Tomsk, Tomsk University Publ. $200 \mathrm{p}$.

Tsvilling M.Ya., 1989. Specific character of social scientific text (about specific differentiation of scientific language). Raznovidnosti $i$ zhanry nauchnoy prozy. Lingvostilisticheskie osobennosti. Moscow, Nauka Publ. pp. 27-36.

Cassitto M., Fattorini E., Gilioni R., Rengo C., 2003. Raising Awareness of Stress at Work in Developing Countries. Protecting Workers: Health Series, no. 6. Milano, World Health Organisation, pp. 1-38. URL: http://www. wascorporation.it/docs/psicological_stress.pdf (accessed February 15, 2018).

Crawshaw L., 2009. Workplace Bullying? Mobbing? Harassment? Distraction by a thousand definitions. Consulting Psychology Journal: Practice and Research, vol. 61, no. 3, pp. 263267. URL: http://www.bosswhispering.com/ CPJ\%20Crawshaw\%20Article.pdf. (accessed 7 July 2017).

\section{SOURCES AND DICTIONARIES}

Druzhilov S.A., 2011. Mobbing at a university's department in the conditions of high school reformation. Vysshee obrazovanie $v$ Rossii [Higher Education in Russia], no. 6, pp. 118-122. URL: http://druzhilov.ru/articles/психологический-террор-боссинг-и-мо/.

Efremova T.F., 2011. Modern explanatory dictionary of the Russian language. URL: http://dic. academic.ru/dic.nsf/efremova/276686/моббинг.

Radchenko I.A., 2007. Dictionary of terms of advertising and public relations. Voronezh, VF MGEI Publ. URL: http://advertising_pr. academic.ru/275/Моббинг.

Breiding M., Basile K., Smith S., Black M., Mahendra R., 2015. Intimate partner violence surveillance: 


\section{МАТЕРИАЛЫ И СООБЩЕНИЯ}

Uniform definitions and recommended data elements, version 2.0. Atlanta, (GA): National Center for Injury Prevention and Control, Centers for Disease Control and Prevention. 156 p. URL: https://www.cdc.gov/violenceprevention/pdf/ intimatepartnerviolence.pdf.

Britannica. URL: https://www.britannica.com/topic/ bullying\#ref1 137234 (accessed 7 July 2017).

Feldman H.R., 2011. Nursing Leadership. A Concise Encyclopedia. URL: http://www.omnilexica. com/?q=mobbing\#noun (accessed 7 July 2017).

Leymann H., 1990. Mobbing and Psychological Terror at Workplaces. Violence and Victims, no. 5, pp. 119-126. URL: http://www.mobbingportal. com/LeymannV\%26V1990(3).pdf (accessed 7 July 2017).

Loveleen K., Varadan S., Kumar P., 2007. Study on Child Abuse: India 2007. Ministry of Women and Child Development, Government of India. 192 p.
Lutgen-Sandvik P., 2003. The Communicative Cycle Of Employee Emotional Abuse. Generation and Regeneration of Workplace Mistreatment. Management Communication Quarterly, vol. 16, no. 4, pp. 471-501. URL: https://www.researchgate. net/publication/258170720_The_Communicative Cycle_Of_Employee_Emotional_Abuse Generation_and_Regeneration_of_Workplace Mistreatment (accessed 20 June 2017).

Mosby's Medical Dictionary, 8th edition. 2009. URL: https://medical-dictionary. thefreedictionary.com/emotional+abuse (accessed 15 February 2018).

Merriam-Webster online dictionary. URL: https:// www.merriam-webster.com/dictionary/mob (accessed 7 July 2017).

Namie G., Yamada D.C. Healthy Workplace Bill. URL: http://healthyworkplacebill.org/problem./ (accessed 15 February 2018).

\section{Information about the Author}

Olga A. Bursina, Candidate of Sciences (Philology), Associate Professor, Department of the English Language, Vologda State University, Lenina St., 15, 160000 Vologda, Russia, olgasazanova@yandex.ru, https://orcid.org/0000-0003-3873-2269

\section{Информация об авторе}

Ольга Алексеевна Бурсина, кандидат филологических наук, доцент кафедры английского языка, Вологодский государственный университет, ул. Ленина, 15, 160000 г. Вологда, Россия, olgasazanova@yandex.ru, https://orcid.org/0000-0003-3873-2269 